\title{
含巴比妥结构荧光探针的合成及其应用研究
}

\author{
刘 钰 ${ }^{a}$ 汤永星 ${ }^{c}$ \\ 罗月阳 ${ }^{c}$ \\ 朱叶婷 $c$ \\ 杨 进 ${ }^{c}$ \\ 魏欣雨 ${ }^{c}$ \\ 刘 雄 ${ }^{c}$ \\ 赵云辉*, $a, b, c$ \\ ( ${ }^{a}$ 河南工业大学小麦和玉米深加工国家工程实验室＼cjkstart郑州 450001) \\ ${ }^{b}$ 中国科学院上海有机化学研究所 天然产物有机合成化学重点实验室 上海 200032) \\ ( ${ }^{c}$ 湖南科技大学化学化工学院 精细聚合物可控制备及功能应用湖南省重点实验室 湘潭 411201)
}

\begin{abstract}
摘要 录离子污染已经严重危害生态环境和人类健康, 发展具有高选择性、高灵敏度且能应用于水性环境中录离子的 检测探针具有重要的研究意义. 以水杨醛为原料, 设计、合成一种含有巴比妥结构单元的激发态分子内质子转移 (ESIPT)型苂光探针; 机理研究预测录离子与探针形成了一种类似于 “(胸腺嘧啶)T-Hg-T” 的结构, 从而可以高选择性 地识别录离子, 并且在 4 20 $\mu \mathrm{mol} \cdot \mathrm{L}^{-1}$ 之间, 探针的荧光强度与录离子浓度呈现非常好的线性关系.

关键词 荧光探针; 录离子; 巴比妥; 激发态分子内质子转移(ESIPT)
\end{abstract}

\section{Synthesis and Application of Fluorescent Probe Containing Barbitone Unit}

\author{
Liu, $\mathrm{Yu}^{a}$ \\ Tang, Yongxing ${ }^{c}$ \\ Luo, Yueyang ${ }^{c}$ \\ Zhu, Yeting $^{c}$ \\ Yang, $\operatorname{Jin}^{c}$ \\ Wei, Xinyu ${ }^{c}$ \\ Liu, Xiong ${ }^{c}$ \\ Zhao, Yunhui ${ }^{*, a, b, c}$ \\ ( ${ }^{a}$ National Engineering Laboratory for Wheat \& Corn Further Processing, Henan University of Technology, Zhengzhou \\ 450001) \\ ( ${ }^{b}$ Key Laboratory of Synthetic Chemistry of Natural Substances, Shanghai Institute of Organic Chemistry, \\ Chinese Academy of Sciences, Shanghai 200032) \\ ( ${ }^{c}$ Hunan Provincial Key Laboratory of Controllable Preparation and Functional Application of Fine Polymers, School of \\ Chemistry and Chemical Engineering, Hunan University of Science and Technology, Xiangtan 411201)
}

\begin{abstract}
Mercury ion pollution has serious harmful impact on the ecological environment and human health. It is of great significance to develop a probe with high selectivity and sensitivity that could be applied to the detection of mercury ion in water environment. In this paper, a novel excited-state intramolecular proton transfer (ESIPT) fluorescent probe containing barbitone unit was designed and synthesized from salicylaldehyde. Mechanism studies showed that mercury ions and probes formed a structure similar to "(thymine)T-Hg-T" which has high selectivity in determining mercury ions. The calibration curve indicated that there was a good linear correlation between the relative fluorescent intensities over the concentration range of $4 \sim 20 \mu \mathrm{mol} \cdot \mathrm{L}^{-1}$ of $\mathrm{Hg}^{2+}$ ion.

Keywords fluorescent probe; mercury ion; barbitone; excited-state intramolecular proton transfer (ESIPT)
\end{abstract}

工业革命在推动社会进步的同时, 也带来了不少的 环境污染问题. 众所周知, 重金属的过度使用与排放已 经成为生态环境和人类健康的一大杀手, 其中录的广泛 存在与使用, 已经引起化学家、生物学家、医学家等的 高度重视. 录元素在自然界中主要以二价化合物或阳离
子形态存在，由于其具有生物难降解性能，因此容易通 过生物链在生物体内不断积聚, 而与生物体内蛋白质和 酶中亲核性较高的硫醇生成硫醇盐, 引起细胞功能性障 碍和与之相关的健康问题 ${ }^{[1,2]}$. 另一方面, 录离子在环境 中遇到细菌，很大程度上会被转变成甲基录，而这种有

\footnotetext{
* Corresponding author. E-mail: zhao_yunhui@163.com

Received April 1, 2019; revised May 5, 2019; published online May 21, 2019.

Project supported by the Open Project of the National Engineering Laboratory for Wheat \& Corn Further Processing, Henan University of Technology (No. NL2018006).

河南工业大学小麦和玉米深加工国家工程实验室开放课题(No. NL2018006)资助项目.
} 
机采会给中枢神经系统造成不可逆转的侵害 ${ }^{[3,4]}$. 因此, 发展快速识别与检测环境和生物体内采离子的有效手 段, 具有非常重要的研究意义与应用价值.

目前, 已经报道能够用于采离子检测的方法有，原

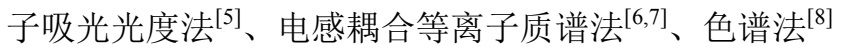
以及电化学分析方法 ${ }^{[9]}$ 等. 其中, 苂光分析法因其灵敏 度高、操作简便、价格低廉的优势成为录离子检测的首

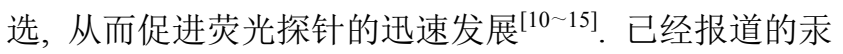
离子检测苂光探针可以分为两大类: 一类是基于配位型 的苂光探针 ${ }^{[16,17]}$; 另一类是反应型的苂光探针 ${ }^{[18 ~ 26]}$. 前 者因为配位作用通常导致探针的荧光猝灭, 后者则因为 发生化学反应使探针产生一种新的苂光物质, 因此体系 的苂光通常是从无到有. 尽管反应型探针具有苂光 “关开” 的特征和优异的选择性, 但是此类探针的水溶性一 般不好, 故能够有效应用于水体系及生物体系录离子检 测的还不多. 基于本课题组在苂光探针方面的研究兴

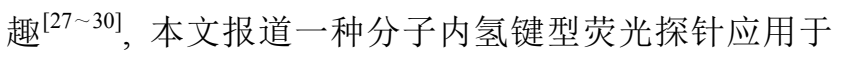
采离子的定性识别与定量检测. 该探针具有两点优势: （1)基于类胸腺嘧啶-巴比妥结构单元对录离子的特异性 结合, 使得探针具有优异的选择性; (2)能够在乙腈 $(\mathrm{MeCN})-$ 水的混合溶液中进行测量, 可以用于环境体系 中采离子的检测研究.

\section{1 结果与讨论}

\section{1 探针分子的设计、合成}

DNA 中的胸腺嘧啶 $(\mathrm{T})$ 能与采离子高度特异性结合 形成稳定的 $\mathrm{T}-\mathrm{Hg}^{2+}-\mathrm{T}$ 配合物, 这一特性使得胸腺嘧啶 成为识别、检测录离子的优良配体 ${ }^{[31,32]}$. 分子内质子转 移型荧光染料是一类非常好的探针, 广泛地应用于各种 离子以及生物分子的识别与检测研究中. 我们设想在 ESIPT 型染料分子 5 上连接巴比妥酸分子, 得到含有类 胸腺嘧啶结构单元的探针分子 6,6 与采离子结合后将引 起苂光强度或最大发射波长的改变, 从而实现对录离子 的定性识别与定量检测.

以水杨醛为原料, 在浓盐酸的作用下与多聚甲醛反 应生成氯甲基化产物 2 ; 化合物 2 在硫酸铜催化下发生 水解反应形成着弪甲基化产物 $\mathbf{3}^{[33]}$; 然后在硝酸银的催化 下与邻氨基苯硫酚发生缩合反应得到苯并噻唑化合物 4; 再经过戴斯马丁(DMP)氧化剂氧化得到 5-甲酰基的 ESIPT 型苂光分子 5; 5 与巴比妥酸分子进行缩合得到目 标探针 6 (Scheme 1). 在制备苯并噻唑化合物 4 的反应 中, 首先尝试使用碘作催化剂在甲醇中进行, 得到的产 物是二氢苯并噻唑衍生物, 中间体没能进一步氧化生成 目标产物; 而更换使用硝酸银/二甲亚砜(DMSO)体系, 硝酸银的用量不到 5\%就可以高达 $90 \%$ 的产率得到中间
体 4.

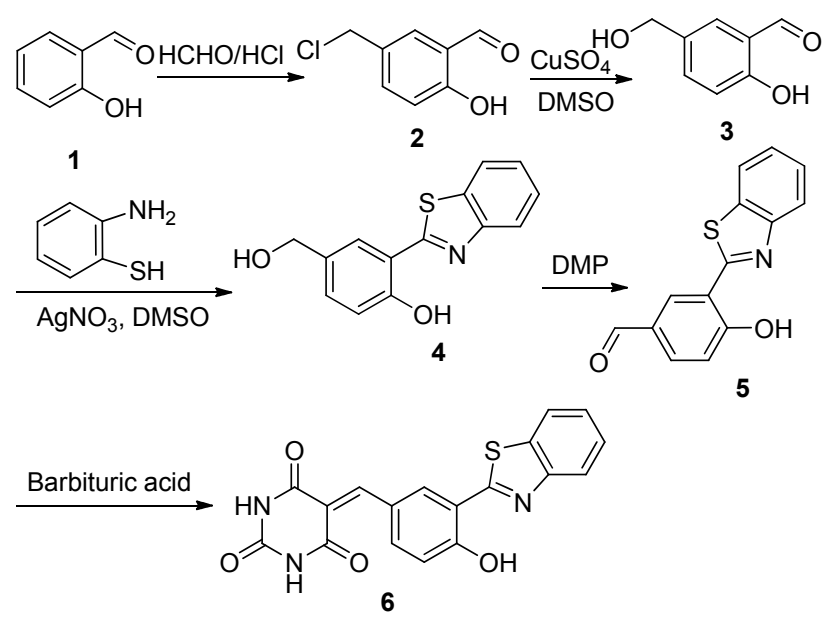

图式 1 探针分子的合成路线

Scheme 1 Synthetic route for probe

\section{2 探针分子的光学性质研究}

相比其它有机溶剂, 探针分子在 $\mathrm{MeCN}$ 中苂光强度 较强, 且最大发射波长也相对较大, 在四氢呋喃(THF) 中几乎没有什么荧光. 在 $\mathrm{MeCN}$ 中, 探针化合物的荧光 相对前体化合物 5 , 最大发射波长从 $444 \mathrm{~nm}$ 红移至 553 $\mathrm{nm}$ 处(图 1), 这主要是因为化合物 5 与巴比妥酸结构单 元发生缩合反应后，母体分子能进一步与两个酰胺键发 生共轭, 使共轭结构得到延长. 并且, 探针分子 6 的苂 光强度相比前体化合物 $\mathbf{5}$ 增强许多, 这种荧光增强是由 于探针分子具有较强的分子内电荷转移现象. 进一步考 察探针在 $\mathrm{MeCN}$ 与水的混合体系中的苂光发射现象: 在 $\mathrm{MeCN}$ 中加入水以后, $380 \mathrm{~nm}$ 激发下苂光的发射波长从 $553 \mathrm{~nm}$ 处蓝移至 $445 \mathrm{~nm}$ 处; 随着水的比例不断增加(体 积比从 $1: 9$ 到 $5: 5$ ), 苂光呈现逐渐增强的趋势; 但是 水的体积比进一步增大到超过 $9: 1$ 时, 体系的苂光又

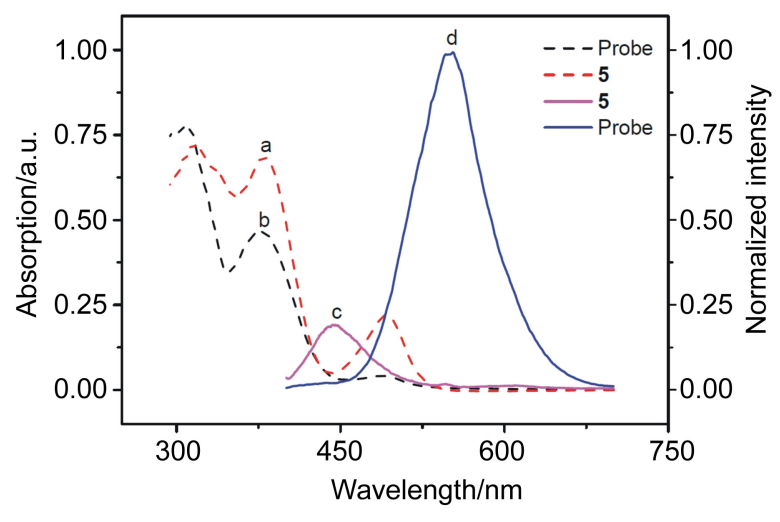

图 1 苂光探针在乙腈中的紫外吸收与菼光光谱

Figure 1 UV-visible, fluorescence spectra of probe in $\mathrm{MeCN}$ (a) UV-visible spectra of 5; (b) UV-visible spectra of probe; (c) fluorescence spectra of 5; (d) fluorescence spectra of probe 
开始逐渐减弱(图 2). 出现这种蓝移现象的原因可能是 由于水分子的存在, 与苂光分子发生了氢键作用, 使苂 光分子的基态振动能级发生了改变. 为便于检测生态环 境中的录离子, $\mathrm{MeCN} / \mathrm{H}_{2} \mathrm{O}$ 体积比为 $5: 5$ 的体系被作 为优选体系用于后续的离子选择性研究以及录离子的 滴定研究中.

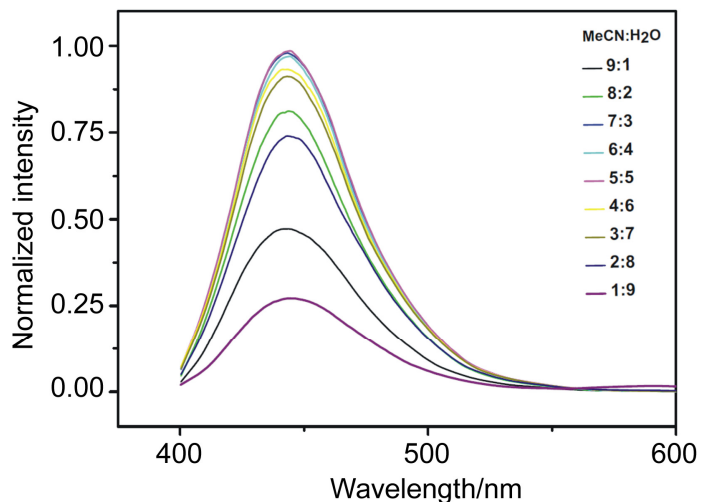

图 2 探针 $\left(10 \mu \mathrm{mol} \cdot \mathrm{L}^{-1}\right)$ 在不同比例 $\mathrm{MeCN} / \mathrm{H}_{2} \mathrm{O}$ 体系中的荧光 光谱

Figure 2 Effect of $\mathrm{MeCN}$ concentration $\left(V_{\mathrm{MeCN}} / V_{\text {water }}=9 / 1,8 / 2\right.$, $7 / 3,6 / 4,5 / 5,4 / 6,3 / 7,2 / 8,1 / 9$, from up to down) on fluorescence intensity of probe $\left(10 \mu \mathrm{mol} \cdot \mathrm{L}^{-1}\right)$

\section{3 探针分子对采离子的定量分析}

探针对录离子的响应速率非常快, 加入采离子 5 $\min$ 以后, 体系的荧光强度几乎不再发生变化. 故荧光 滴定在体系加入采离子摇匀静置 $5 \mathrm{~min}$ 后进行, 测定结 果如图 3 所示. 加入采离子以后, 体系的荧光强度随求 离子浓度 $\left(0 \sim 20 \mu \mathrm{mol} \cdot \mathrm{L}^{-1}\right)$ 的增加而下降, 但荧光最大 发射波长基本没有什么变化, 说明络合过程对苂光染料 的共轭结构的影响可以忽略不计. 从图 $3 \mathrm{~b}$ 可知, 采离子 浓度在 $4 \sim 20 \mu \mathrm{mol} \cdot \mathrm{L}^{-1}$ 内与 $\Delta F / F_{0}$ 之间存在非常好的线 性关系 $\Delta F / F_{0}=0.0294 c-0.0806\left(R^{2}=0.997\right)$, 检出限 (LOD)为 $2.74 \mu \mathrm{mol} \cdot \mathrm{L}^{-1}$. 其中 $F_{0}$ 为不加录离子时的苂光 强度, $F$ 为添加采离子后溶液体系的苂光, $\Delta F=F_{0}-F$.

\section{4 探针分子对金属离子的选择性}

胸腺嘧啶对录离子的特异性结合使其成为录离子 识别的优选配体，该探针分子含有类似胸腺嘧啶的结构 单元. 为了验证最初对该分子的设想, 我们考察了该探 针对一系列金属离子的选择性. 在前面优化的实验条件 下, 将 $10 \mu \mathrm{mol} \cdot \mathrm{L}^{-1}$ 的 $\mathrm{Hg}^{2+} 、 \mathrm{Sr}^{2+} 、 \mathrm{Cd}^{2+} 、 \mathrm{Na}^{+} 、 \mathrm{Li}^{+}$、 $\mathrm{Ca}^{2+} 、 \mathrm{~Pb}^{2+} 、 \mathrm{~K}^{+} 、 \mathrm{Zn}^{2+} 、 \mathrm{Mg}^{2+} 、 \mathrm{Co}^{2+} 、 \mathrm{Al}^{3+} 、 \mathrm{Fe}^{3+} 、$ $\mathrm{Fe}^{2+} 、 \mathrm{Ba}^{2+} 、 \mathrm{Pd}^{2+} 、 \mathrm{Ag}^{+} 、 \mathrm{Cr}^{2+} 、 \mathrm{Cu}^{2+} 、 \mathrm{Ni}^{2+}$ 等分别加入 到 $10 \mu \mathrm{mol} \cdot \mathrm{L}^{-1}$ 的探针溶液中, 然后在 $380 \mathrm{~nm}$ 的激发下, 测定各体系的苂光发射情况. 从图 $4 \mathrm{a}$ 可以看出, 只有录
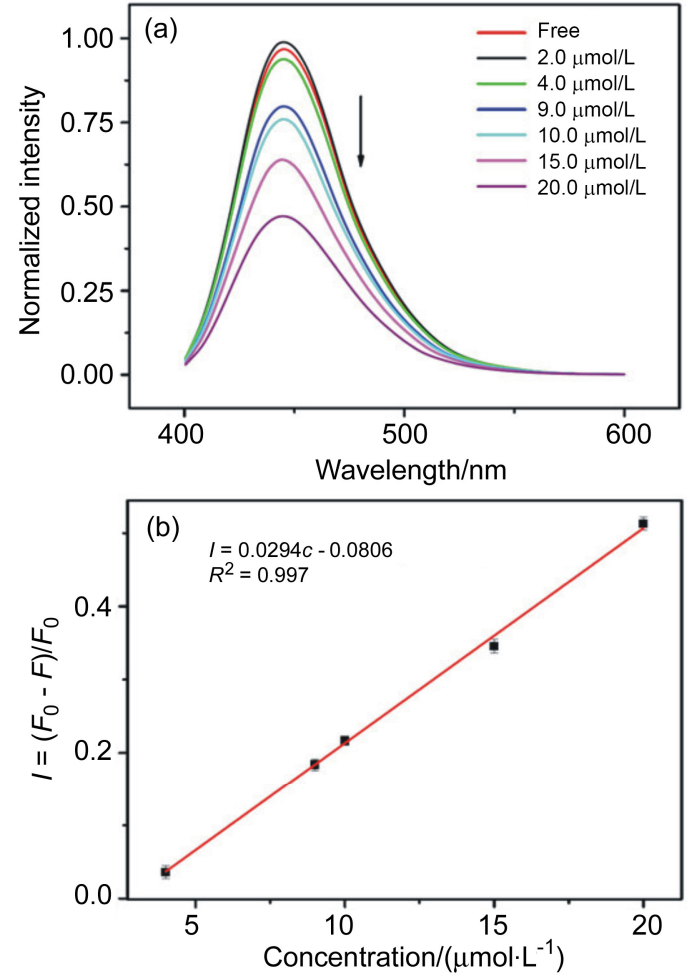

图 3 (a)探针分子 $\left(10 \mu \mathrm{mol} \cdot \mathrm{L}^{-1}\right)$ 加入 $\mathrm{Hg}^{2+}(2.0,4.0,9.0,10.0$, $15.0,20.0 \mu \mathrm{mol} \cdot \mathrm{L}^{-1}$, 从上到下)后苂光强度的变化以及(b)荧光 强度变化 $\Delta F / F_{0}$ 对 $\mathrm{Hg}^{2+}$ 浓度的线性关系

Figure 3 (a) Fluorescent emission changes of probe $(10 \mu \mathrm{mol}$ $\left.\mathrm{L}^{-1}\right)$ in the presence of increasing amounts of $\mathrm{Hg}^{2+}(0,2.0,4.0$, $9.0,10.0,15.0,20.0 \mu \mathrm{mol} \cdot \mathrm{L}^{-1}$, from up to down) in $50 \%$ $\mathrm{MeCN}-\mathrm{H}_{2} \mathrm{O}(V / V)$, and (b) the linear relationship between the relative fluorescent intensities $\left(\Delta F / F_{0}\right)$ and concentration of $\mathrm{Hg}^{2+}$

离子对探针的苂光具有非常明显的猝灭作用，而其它金 属阳离子对体系的苂光影响甚微，反映探针对录离子的 结合具有非常好的专一性. 在已有的研究报道中, $\mathrm{Cd}^{2+}$ 、 $\mathrm{Pb}^{2+} 、 \mathrm{Cu}^{2+} 、 \mathrm{Zn}^{2+}$ 和 $\mathrm{Ag}^{+}$等金属阳离子的存在可能会对 永离子的检测产生干扰作用, 降低探针的专一性. 因此, 本研究将探针中同时加入干扰离子和录离子与只加入 相应干扰离子的两个体系的荧光强度进行对比，研究发 现干扰离子对录离子检测的影响几乎可以忽略(图 4b).

\section{5 探针分子对录离子配位机理分析}

为了研究探针分子对录离子的识别机理, 将探针与 等物质的量的 $\mathrm{NaOH}$ 溶液混合, 体系的荧光几乎消失不 见(图 5). 这种现象说明两个问题: (1)该探针是一种 ESIPT 型的苂光探针，因为荧光分子酚羟基上的质子被 碱夺取以后，体系在激发态下不能产生分子内质子转移 现象，从而导致荧光基本消失; (2)录离子与探针的巴比 妥酸结构单元形成了类 “T-Hg-T” 的结构. 当 2 equiv. 的录离子与探针作用后，苂光还比较强，说明录离子不 是通过破坏探针分子内氢键来配位的，而是通过巴比妥 

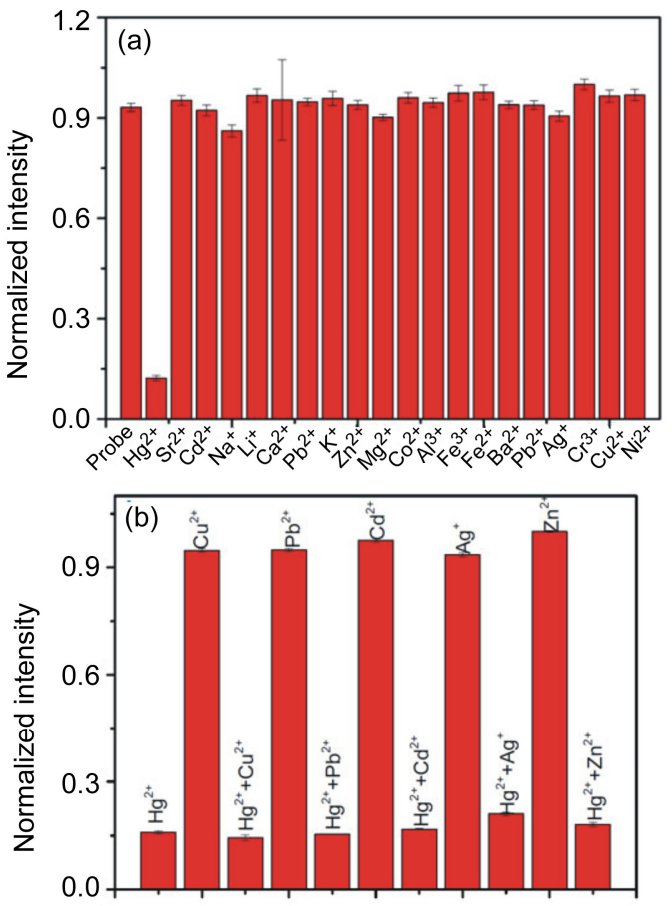

图 4 (a)探针 $\left(10 \mu \mathrm{mol} \cdot \mathrm{L}^{-1}\right)$ 中分别加入各种阳离子 $(10 \mu \mathrm{mol} \cdot$ $\left.\mathrm{L}^{-1}\right)$ 的荧光强度变化柱状图, 以及 $(\mathrm{b})$ 探针 $\left(10 \mu \mathrm{mol} \cdot \mathrm{L}^{-1}\right)$ 中加入 录离子和其它阳离子 $\left.(10 \mu \mathrm{mol} \mathrm{L})^{-1}\right)$ 的苂光强度变化柱状图

Figure 4 Fluorescent emission changes of probe $\left(10 \mu \mathrm{mol} \mathrm{L}^{-1}\right)$ upon addition of various cations $\left(10 \mu \mathrm{mol} \cdot \mathrm{L}^{-1}\right)$ in $50 \% \mathrm{H}_{2} \mathrm{O}-$ $\mathrm{MeCN}$ solution (a) and fluorescence spectra of probe (10 $\left.\mu \mathrm{mol} \cdot \mathrm{L}^{-1}\right)$ in the presence of $\mathrm{Hg}^{2+}\left(10 \mu \mathrm{mol} \cdot \mathrm{L}^{-1}\right)$ and other metal ions $\left(10 \mu \mathrm{mol} \cdot \mathrm{L}^{-1}\right)(\mathrm{b})$

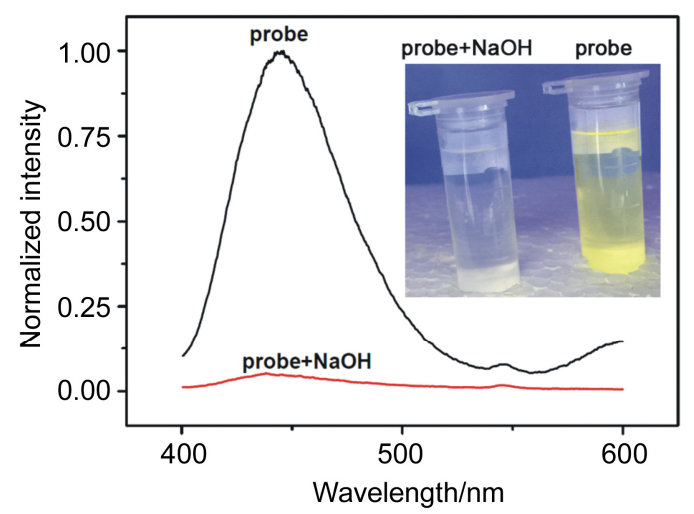

图 5 探针分子 $\left(10 \mu \mathrm{mol} \cdot \mathrm{L}^{-1}\right)$ 、探针分子 $\left(10 \mu \mathrm{mol} \cdot \mathrm{L}^{-1}\right)$ 体系中 加入等当量的 $\mathrm{NaOH}$ 后的苂光光谱

Figure 5 Fluorescent emission of probe $\left(10 \mu \mathrm{mol} \cdot \mathrm{L}^{-1}\right)$ and the system of probe $\left(10 \mu \mathrm{mol} \cdot \mathrm{L}^{-1}\right)$ mixed with $\mathrm{NaOH}\left(10 \mu \mathrm{mol} \cdot \mathrm{L}^{-1}\right)$

酸结构单元中的 $\mathrm{N}$ 与 $\mathrm{Hg}^{2+}$ 结合, 又并不是正好两分子 的探针与 1 个 $\mathrm{Hg}^{2+}$ 结合, 因为当 $\mathrm{Hg}^{2+}$ 达到 2 equiv. 的时 候, 探针的苂光强度与录离子浓度还能形成较好的线性 关系. 这也就预示着巴比妥酸结构中的两个 $\mathrm{N}$ 原子均有 机会参与录离子配位, 与所测定的 Job 曲线不出现明显 拐点的现象相符.

\section{2 结论}

设计、合成了一种类似于胸腺嘧啶结构的苂光探针, 并利用核磁共振、红外光谱和高分辨质谱对目标化合物 进行结构确认．通过光学研究发现，该探针分子在乙腈 中有比较好的荧光发射, 且在 $\mathrm{MeCN} /$ 水的体积比为 5 : 5 时苂光发射最强. 苂光滴定表明在采离子浓度为 4 $20 \mu \mathrm{mol} \cdot \mathrm{L}^{-1}$ 之间, 探针荧光强度 $\Delta F / F_{0}$ 与采离子浓度有 非常好的线性关系. 进一步的离子选择研究显示, 探针 对录离子具有特异性结合的优势，对其它离子几乎没有 响应. 机理研究表明探针分子可能是通过巴比妥酸结构 单元中的 $\mathrm{N}$ 与采离子结合. 该研究的进行为录离子的识 别、检测研究提供了一种新思路.

\section{3 实验部分}

\section{1 仪器与试剂}

熔点用 Yanaco MP500 型显微熔点测定仪(温度未校 正); 高分辨质谱在液相色谱/质谱联用仪 Xevo Q-Tof MS 上测定; IR 光谱在 Nicolet 5-DX 型 FT-IR 红外光谱 仪上测定 $(\mathrm{KBr}$ 压片); 核磁共振在 Bruker AV-II $400 \mathrm{MHz}$ NMR 核磁共振仪上测定, 以 TMS 为内标, 氝代二甲亚 砜为溶剂. 所用药品均为市售的分析纯或化学纯, 除特 别注明外, 未经进一步处理.

\section{2 实验方法}

\subsubsection{5-氯甲基水杨醛(2)的合成}

该化合物根据已知文献[33]合成报道. 将多聚甲醛 $(2.7 \mathrm{~g}, 90 \mathrm{mmol})$ 、水杨醛(12.2 g, $100 \mathrm{mmol})$ 加入到 100 $\mathrm{mL}$ 的浓盐酸中, 反应体系在 $70{ }^{\circ} \mathrm{C}$ 下搅拌 $24 \mathrm{~h}$. 反应结 束后, 沉淀过滤并用蒸馏水清洗, 重新溶于乙酸乙酯干 燥. 溶剂减压旋蒸除去, 粗产物通过石油醚重结晶得到 白色固体，产率 $36 \%$.

\subsubsection{5-羟甲基水杨醛 $(3)$ 的合成}

化合物 2 (1.7 g, $10 \mathrm{mmol})$ 和五水硫酸铜 $(2.5 \mathrm{~g}, 10$ $\mathrm{mmol})$ 加入到二甲亚砜(DMSO) $(15 \mathrm{~mL})$ 和水 $(7 \mathrm{~mL})$ 的混 合溶剂中, 反应体系在 $110{ }^{\circ} \mathrm{C}$ 下搅拌 $2 \mathrm{~h}$, 然后冷却至 室温, 用水稀释, 然后乙酸乙酯萃取, 再用水和饱和食 盐水洗涤, 无水硫酸钠干燥. 减压旋蒸除去乙酸乙酯即 得中间体 $\mathbf{3}^{[33]}$.

\subsubsection{2-(苯并噻唑-2-)-4-羟甲基苯酚(4)的合成}

邻氨基苯硫酚(112 mg, $1.1 \mathrm{mmol}), 5$-差圣甲基水杨醛 (3) (152 mg, $1 \mathrm{mmol}$ )依次加入到 $3 \mathrm{~mL}$ DMSO 中, 然后 将硝酸银 $(8 \mathrm{mg}, 5 \mathrm{~mol} \%$ )加入反应液中, 在室温下搅拌 至原料消失, 用乙酸乙酯稀释反应液, 然后加入 $30 \mathrm{~mL}$ 水, 用乙酸乙酯萃取 $(15 \mathrm{~mL} \times 3)$, 蒸馏水与饱和食盐水 各洗涤 2 次, 无水硫酸钠干燥, 旋干得到粗产物, 无需 
纯化直接进行下一步反应.

\subsubsection{3-(苯并噻唑-2-)-4-羟基苯甲醛(5)的合成}

2-苯并噻唑-4-羟甲基苯酚(510 mg, $2 \mathrm{mmol}$ )溶于 20 $\mathrm{mL}$ 的无水二氯甲烷中, 然后加入 DMP (Dess-Martin Periodinane) (1.27g, $3 \mathrm{mmol}$ ), 室温反应 $2 \mathrm{~h}$, 原料全部消 失, 加水淬灭反应, 然后用二氯甲烷萃取, 无水硫酸钠 干燥, 旋干除去有机溶剂得到粗产物, 柱层析 $[V($ 石油 醚) $: V$ (乙酸乙酯 $)=5 ： 1$ ] 得到纯净产物 $\mathbf{5}^{[34]}$, 产率 $85 \%$. 3.2.5 5-(苯并噻唑-2-)-4-羟芐叉嘧啶-2,4,6 (1H,3 $H$, $5 H)$-三酮 $(6)$ 的合成

化合物 5 (102 mg, $0.4 \mathrm{mmol})$ 和巴比妥酸 $(52.5 \mathrm{mg}$, $0.41 \mathrm{mmol}$ )加入到 $8 \mathrm{~mL}$ 无水乙醇中, 反应体系在 $80{ }^{\circ} \mathrm{C}$ 下搅拌 $4 \mathrm{~h}$, 有大量的淡黄色固体析出, 固体过滤, 然后 用无水乙醇洗涤 $(5 \mathrm{~mL} \times 3)$, 得到目标产物 6 (130 mg), 产率 $89 \%$. 浅黄色固体, 熔点大于 $300{ }^{\circ} \mathrm{C},{ }^{1} \mathrm{H}$ NMR (400 MHz, DMSO- $\left.d_{6}\right) \delta$ : $12.71(\mathrm{br}, 1 \mathrm{H}), 11.36(\mathrm{~s}, 1 \mathrm{H}), 11.26$ (s, $1 \mathrm{H}), 9.38(\mathrm{~d}, J=2.0 \mathrm{~Hz}, 1 \mathrm{H}), 8.35 \sim 8.32(\mathrm{~m}, 2 \mathrm{H}), 8.20(\mathrm{~d}$, $J=8.0 \mathrm{~Hz}, 1 \mathrm{H}), 8.11(\mathrm{~d}, J=8.0 \mathrm{~Hz}, 1 \mathrm{H}), 7.58$ (t, $J=7.2$ $\mathrm{Hz}, 1 \mathrm{H}), 7.48$ (t, $J=7.2 \mathrm{~Hz}, 1 \mathrm{H}), 7.20$ (d, $J=8.8 \mathrm{~Hz}, 1 \mathrm{H})$. ${ }^{13} \mathrm{C}$ NMR $\left(100 \mathrm{MHz} \mathrm{CDCl}_{3}\right) \delta: 164.6,164.2,162.7,160.8$, $154.7,151.7,150.7,139.7,136.4,134.8,127.1,125.8$, 124.8, 122.7, 122.6, 118.8, 117.3, 116.7; IR (KBr ) v: 3498, 3216, 3064, 2856, 1752, 1706, 1656, 1537, 1504, 1478, 1400, 1322, 1222, 1187, 824, $757 \mathrm{~cm}^{-1}$. HRMS calcd for $\mathrm{C}_{18} \mathrm{H}_{12} \mathrm{~N}_{3} \mathrm{O}_{4} \mathrm{~S}[\mathrm{M}+\mathrm{H}]^{+}$366.0549, found 366.0543.

\section{2 .6 荧光光谱法测定录离子的浓度}

探针配成 $10^{-4} \mathrm{~mol} \cdot \mathrm{L}^{-1}$ 的乙腈溶液, 采离子和其它 金属离子配制成 $10^{-3} \mathrm{~mol} \cdot \mathrm{L}^{-1}$ 的水溶液. 取 $0.4 \mathrm{~mL}$ 的探 针溶液加入到 $5 \mathrm{~mL}$ 的样品管中, 再加 $1.6 \mathrm{~mL}$ 乙腈, 然 后取合适量的离子溶液加入到样品管中, 最后用二次蒸 馏水稀释至 $4 \mathrm{~mL}$. 荧光测试在 $380 \mathrm{~nm}$ 激发下, 收集 $400 \sim 600 \mathrm{~nm}$ 区间的发射峰.

辅助材料(Supporting Information) 所有新化合物的 ${ }^{1} \mathrm{H} N M R 、{ }^{13} \mathrm{C}$ NMR、IR、HRMS 图谱. 这些材料可以 免费从本刊网站(http://sioc-journal.cn/)上下载.

\section{References}

[1] Choi, M. G.; Im, H. G.; Noh, J. H.; Ryu, D. H.; Chang, S. K. Sens. Actuators, B 2013, 177, 583 .

[2] Zhang, D.; Su, J. H.; Ma, S.; Tian, H. Tetrahedron 2008, 64, 8515.

[3] Zalups, R. K.; Ahmad, S. J. Am. Soc. Nephrol. 2004, 15, 2023.

[4] Silbergeld, E. K.; Silva, I. A.; Nyland, J. F. Toxicol. Appl. Pharmacol. 2005, 207, 282
[5] Tseng, C. M.; Diego, A. D.; Martin, O. F.; Amouroux, D.; Donard, O. F. J. Anal. At. Spectrom. 1997, 12, 743.

[6] Chen, F.; Li, Y.; Li, B.; Sun, J.; Wang, X.; Gao, X. J. Anal. At. Spectrom. 2006, 21, 94.

[7] Qian G. S.; Zhao, W.; Xu, J. J. Tao, H. Y. Acta Chim. Sinica 2017, 75, 1097 (In Chinese). (钱广盛, 赵微, 徐静娟, 陈洪渊, 化学学报, 2017, 75, 1097.)

[8] Nolan, E. M.; Lippard, S. J. Chem. Rev. 2008, 108, 3443.

[9] Xuan, F.; Luo, X.; Hsing, I. M. Anal. Chem. 2013, 85, 4586.

[10] Li, Y.; Shi, W.; Ma, J.; Wang, X.; Kong, X.; Zhang, Y.; Feng, L.; Hui, Y.; Xie, Z. J. Photochem. Photobiol., A 2017, 338, 1

[11] Feng, L.; Shi, W.; Ma, J.; Chen, Y.; Fan, K.; Hui, Y.; Xie, Z. Sens. Actuators, B 2016, 237, 563.

[12] Lin, H.; Shi, W.; Tian, Y.; Ma, F.; Xu, L.; Ma, J.; Hui, Y.; Xie, Z. J. Lumin. 2015, 157, 280.

[13] Kim, H. N.; Ren, W. X.; Kim, J. S.; Yoon, J. Chem. Soc. Rev. 2012, $41,3210$.

[14] Wang, H.; Zhang, P.; Chen, J.; Li, Y.; Yu, M.; Long, Y.; Yi, P. Sens. Actuators, B 2017, 242, 818.

[15] Fan, Y.; Long, Y. F.; Li, Y. F. Anal. Chim. Acta 2009, 653, 207

[16] Zhu, J. H.; Fan, X. T.; Jiang, P.; Cao, Q. Y. Chin. J. Org. Chem. 2017, 37, 185 (in Chinese). (朱江华, 范香田, 蒋平, 曹迁永, 有机化学, 2017, 37, 185.)

[17] Shiraishi, Y.; Sumiya, S.; Hirat, T. Org. Biomol. Chem. 2010, 8, 1310.

[18] Zhang, Y.; Wang, Q.; Li, W.; Zhang, J.; Xia, M. H.; Chen, S. M.; Chen, X. M. Chin. J. Org. Chem. 2014, 34, 403 (in Chinese). (张勇, 王强, 李伟, 张静, 夏明辉, 陈世明, 陈雪梅, 有机化学, 2014, 34, 403.)

[19] Yang, Y. K.; Yook, K. J.; Tae, J. A. J. Am. Chem. Soc. 2005, 127, 16760 .

[20] Chen, J.; Li, Y.; Zhong, W.; Wang, H.; Zhang, P.; Jiang. J. Anal. Methods 2016, 8, 1964.

[21] Zhang, D.; Li, M.; Jiang, Y.; Wang, C.; Wang, Z.; Ye, Y.; Zhao, Y. Dyes Pigm. 2013, 99, 607.

[22] Wang, C.; Zhang, D.; Huang, X.; Ding, P.; Wang, Z.; Zhao, Y.; Ye, Y. Sens. Actuators, B 2014, 198, 33.

[23] Ma, S.; Li, L.; She, M.; Mo, Y.; Zhang, S.; Liu, P.; Li, J. Chin. Chem. Lett. 2017, 28, 2014.

[24] Song, J.; Huai, M.; Wang, C.; Xu, Z.; Zhao, Y.; Ye, Y. Spectrochim. Acta, Part A 2015, 139, 549

[25] Yang, L.; Su, Y.; Geng, Y.; Xiong, H.; Han, J.; Fang, Q.; Song, X. Org. Biomol. Chem. 2018, 16, 5036.

[26] Zhang, P. P.; Chen, S.; Kang, Y. F.; Long, Y. F. Spectrochim. Acta, Part A 2012, 99, 347.

[27] Zhao, Y.-H.; Luo, Y.; Wang, H.; Guo, T.; Zhou, H.; Tan, H.; Zhou, Z.; Long, Y.; Tang, Z. ChemistrySelect 2018, 3, 1521.

[28] Zhao, Y.-H.; Li, Y.; Long, Y.; Zhou, Z.; Tang, Z.; Deng, K.; Zhang, S. Tetrahedron Lett. 2017, 58, 1351.

[29] Zhao, Y.-H.; Luo, Y.; Wang, H.; Wei, H.; Guo, T.; Tan, H.; Yuan, L.; Zhang, X.-B. Anal. Chim. Acta 2019, 1065, 134.

[30] Zhao, Y.-H.; Luo, Y.; Guo, T.; Tang, Z.; Zhou, Z. ChemistrySelect 2019, 4,5195

[31] Tanaka, Y.; Oda, S.; Yamaguchi, H.; Kondo, Y.; Kojima, C.; Ono, A. J. Am. Chem. Soc. 2007, 129, 244.

[32] Hou, P.; Long, Y.; Zhao, J.; Wang, J.; Zhou, F. Spectrochim. Acta, Part $A$ 2012, 86, 76 .

[33] Sfrazzetto, G. T.; Millesi, S.; Pappalardo, A.; Tomaselli, G. A.; Ballistreri, F. P.; Toscano, R. M.; Fragalà, I.; Gulino, A. Chem.-Eur. J. 2017, 23, 1576.

[34] He, X.; Wu, Y.; Jin, W.; Wang, X.; Wu, C.; Shang, Y. Appl. Organomet. Chem. 2018, 32, e4284. 